\title{
miR-125b inhibited epithelial-mesenchymal transition of triple-negative breast cancer by targeting MAP2K7
}

\author{
Liquan Hong' \\ Feng Pan' \\ Huifen Jiang ${ }^{2}$ \\ Lahong Zhang' \\ Yuhua Liu' \\ Chengsong Cai' \\ Chunzhen $\mathrm{Hua}^{3}$ \\ Xian Luo' \\ Jinhua Sun ${ }^{4}$ \\ Zhaojun Chen' \\ 'Department of Clinical Laboratory, \\ Affiliated Hospital of Hangzhou \\ Normal University, ${ }^{2} Z$ hejiang \\ Provincial Tumor Hospital, ${ }^{3}$ Zhejiang \\ Provincial Children's Hospital, \\ ${ }^{4}$ Technology Department, Hangzhou \\ Joingenome Diagnostics, Hangzhou, \\ Zhejiang Province, People's Republic \\ of China
}

Correspondence: Zhaojun Chen Department of Clinical Laboratory, Affiliated Hospital of Hangzhou Normal University, 126 Wenzhou Road, Hangzhou, Zhejiang Province 3/I200,

People's Republic of China

Tel/fax +86571883580 II

Email chenzhaojunI5@I63.com
This article was published in the following Dove Press journal:

OncoTargets and Therapy

4 May 2016

Number of times this article has been viewed
Abstract: MicroRNAs (miRNAs) play important roles in diverse biological processes and are emerging as key regulators of tumorigenesis and tumor progression. Among the differentially expressed miRNAs in breast cancer, miR-125b was revealed to be deregulated and associated with poor prognosis and chemoresistance in triple-negative breast cancer (TNBC), but the mechanism is still unknown. In our study, we showed downregulated expression of miR-125b in TNBC tissues and decreased migration and invasion in miR-125b-expressing Hs578T cells. MAP2K7 was then detected to be a novel target of miR-125b, and downregulation of $M A P 2 K 7$ by miR$125 \mathrm{~b}$ was similar to transient knockdown of $M A P 2 K 7$ which hindered epithelial-mesenchymal transition (EMT) of Hs578T cells. Upregulation of MAP2K7 in miR-125b-overexpressing Hs $578 \mathrm{~T}$ cells partly rescued the migration and invasion suppression of miR-125b. Furthermore, MAP2K7 was overexpressed in TNBC samples compared with normal tissues and negatively correlated with miR-125b expression. In light of these findings, miR-125b emerged as a tumor suppressor in TNBC by targeting MAP $2 K 7$ to inhibit EMT.

Keywords: miR-125b, MAP2K7, TNBC, Hs578T, EMT

\section{Introduction}

Breast cancer is the most common type of cancer in women worldwide. ${ }^{1}$ Approximately, 1.2 million new cases and 465,000 deaths occur each year, accounting for $23 \%$ of total cancer cases and $14 \%$ of cancer deaths, and its incidence is increasing in many countries including the People's Republic of China. ${ }^{2,3}$ Importantly, triple-negative breast cancer (TNBC) is the most invasive and aggressive among the breast cancer subtypes. ${ }^{4}$ TNBC is typically of the histological ductal type, characterized by a lack of expression of $\alpha$-estrogen, progesterone, and HER 2 receptors; high mitotic rate; increased lymphocytic infiltrate; and high grade and large tumor size., ${ }^{5,6}$ addition, TNBC patients commonly present with early visceral metastases and have lymph node involvement at the time of diagnosis. Approximately $15 \%$ of TNBC patients will develop brain metastases while autopsy studies report a rate of $30 \% .{ }^{6}$ So far, there is no clinical therapy specific for patients with TNBC. ${ }^{4}$ Therefore, identifying biological markers of TNBC progression could be meaningful for the prevention of breast cancer metastasis and providing new therapeutic strategies for the disease.

MicroRNAs (miRNAs) are a class of endogenous noncoding single-stranded RNAs $\sim 22$ nucleotides (nt) in length, which regulate amounts of protein expressed from coding RNAs by translational repression or by cleavage of the target mRNA due to base pairing with the $3^{\prime}$-untranslated region (UTR). ${ }^{7}$ miRNAs have been linked to essential 
physiologic processes such as proliferation, differentiation, and apoptosis, and to several diseases including cancer. ${ }^{7-10}$ Over recent years, miRNA expression studies, especially large-scale profiling, have provided evidence that the aberrant expression of miRNA is associated with human breast cancer. ${ }^{11}$ Among the differentially expressed miRNAs in breast cancer, miR-125b was revealed to be deregulated, which is associated with poor prognosis and chemoresistance in TNBC, ${ }^{12}$ but the mechanism involved was not clear, so our study focused on determining the effect of miR-125b on TNBC.

The human genome may contain up to 1,000 miRNAs, and $30 \%$ of human mRNAs are predicted to be targets of miRNAs. ${ }^{13}$ In our study, we identified $M A P 2 K 7$ as the main target of miR-125b. $M A P 2 K 7$, also known as $M K K 7$, and MKK4 are kinases that are implicated in the activation of the c-Jun $\mathrm{NH}_{2}$-terminal kinase (JNK) group of MAPK. ${ }^{14}$ They play a nonredundant role in the dual phosphorylation of JNK at Thr183 and Tyr185 (P-JNK). ${ }^{15}$ MKK4 also activates p38, whereas MAP2K7 specifically activates JNK which, in turn, activates substrates such as transcription factors or proapoptotic proteins. ${ }^{16}$ Moreover, various studies have demonstrated that MAP2K7 is indispensable for JNK activation. ${ }^{17}$

In the present study, downregulation of miR-125b was found in TNBC tissues, so we investigated whether miR-125b may be involved in the inhibition of epithelial-mesenchymal transition (EMT) in TNBC cells. We looked for unknown target genes of miR-125b and identified $M A P 2 K 7$ as a novel target which reversed the inhibitory effect of miR-125b on the EMT of TNBC cells. In addition, MAP $2 K 7$ was upregulated in TNBC specimens and negatively correlated with miR-125b expression.

\section{Materials and methods Clinical specimens}

TNBC and adjacent normal breast tissues were anonymously collected from Affiliated Hospital of Hangzhou Normal University. Written informed consent was obtained from each patient. All of the procedures performed in this study were approved by the Ethics Committee of Affiliated Hospital of Hangzhou Normal University.

\section{Cell culture}

HEK-293T and TNBC cell line Hs578T were purchased from Chinese Academy of Science Cell Bank (Shanghai, People's Republic of China). HEK-293T and Hs578T were cultured in Dulbecco's Modified Eagle's Medium (DMEM; HyClone, Logan, UT, USA) with 10\% fetal bovine serum (FBS). All media contained $1 \%$ penicillin/streptomycin. All cell lines were incubated at $37^{\circ} \mathrm{C}$ with $5 \% \mathrm{CO}_{2}$.

\section{Plasmid construction}

The open reading frame of $M A P 2 K 7$ gene without 3 '-UTR was amplified by polymerase chain reaction (PCR) using the following primers: MAP2K7 Forward (Xhol I): 5'-CTACTC GAGGCAGTGCGGTGTTTGTCTG-3', Reverse (Bam HI): 5'-CTAGGATCCCCCCACTCTCTGTCCTCAGT-3', and then cloned into the pcDNA3.1(-) vector; the recombinant plasmid was named MAP2K7-pcDNA3.1(-).

\section{Transfection}

For miRNA and siRNA, cells were seeded at $\sim 50 \%$ confluence into six-well plate and transiently transfected with miR-125b or scrambled miRNA/siMAP2K7 or scrambled siRNA (GenePharma Co., Ltd, Shanghai, People's Republic of China) to a final concentration of $50 \mathrm{nM}$ using $3 \mu \mathrm{L}$ Lipofectamine 2000 transfection reagent (Thermo Fisher Scientific, Waltham, MA, USA, USA) following the manufacturer's instruction.

For plasmid, cells were seeded at $\sim 80 \%$ confluence into six-well plate and transfected with $2 \mu \mathrm{g} M A P 2 K 7$ pcDNA3.1(-) or pcDNA3.1(-) using $4 \mu \mathrm{L}$ Lipofectamine 2000 transfection reagent.

\section{Dual luciferase assay}

The human MAP2K7 3'-UTR target site was amplified by PCR using the following primers: MAP $2 K 7-3^{\prime} \mathrm{UTR}$ F: 5'-CCGCTCGAGCGATCAGAGTCGCTGTTCATT-3', R: 5'-ATAAGAATGCGGCCGCCACCACAAGCAGCTAA TCG-3' and cloned downstream of the Renilla luciferase gene in psiCHECK-2 vector (Promega Corporation, Fitchburg, WI, USA), which also contains Firefly luciferase gene that can be used as a reference.

The dual luciferase experiment was performed in HEK293 T cells. HEK-293T cells were cultured in six-well plates at $80 \%$ confluence and cotransfected with $2 \mu \mathrm{g}$ constructed plasmid together with $70 \mathrm{nM}$ miR-125b mimics or a scrambled miRNA using $4 \mu \mathrm{L}$ Lipofectamine 2000 reagent according to the manufacturer's protocol. Cells were lysed and assayed for luciferase activity at 48 hours after transfection using a Dual-Luciferase Assay kit (E1910; Promega). Changes in the expression of Renilla luciferase were normalized relative to Firefly luciferase.

\section{RNA isolation, reverse transcription, and quantitative real-time PCR}

Total RNA from patient tissue samples and cell lines was extracted by TRIzol according to the manufacturer's protocol (Thermo Fisher Scientific). The concentration of all RNA samples was quantified using NanoDrop 1000 (Thermo 
Fisher Scientific). The expression of mature miRNAs was assayed using a MicroRNA Reverse Transcription Kit (Promega). Briefly, $12 \mu \mathrm{L}$ of a mixture containing $1 \mu \mathrm{g}$ RNA and diethyl pyrocarbonate-treated $\mathrm{H}_{2} \mathrm{O}$ was held at $65^{\circ} \mathrm{C}$ for 3 minutes and then put on ice immediately. Then, $4 \mu \mathrm{L}$ of $5 \mathrm{X}$ buffer, $0.5 \mu \mathrm{L}$ reverse transcriptase extract from Moloney Murine Leukemia Virus, $0.5 \mu \mathrm{L}$ RNasin, $2 \mu \mathrm{L}$ deoxy-ribonucleoside triphosphates, $0.5 \mu \mathrm{L}$ specific stemloop reverse transcription (RT) primer, and $0.5 \mu \mathrm{L}$ U6 RT were added into the mixture. The reaction mixtures $(20 \mu \mathrm{L})$ were incubated as follows: $20^{\circ} \mathrm{C}$ for 10 minutes, $42^{\circ} \mathrm{C}$ for 50 minutes, $75^{\circ} \mathrm{C}$ for 5 minutes, and held at $4^{\circ} \mathrm{C}$.

Reverse transcription of miR-125b or endogenous control of the small nuclear U6 was performed by using total RNA with stem-loop RT-specific primers, and the sequences were as follows: miR-125b: 5'-CTCAACTGGTGTCG TGGAGTCGGCAATTCAGTTGAGTCACAAGTT-3'; U6: 5'-CGCTTCACGAATTTGCGTGTCAT-3'. Reverse transcription of $M A P 2 K 7$ was performed using a PrimeScript RT Reagent Kit (RR037A; Takara Bio Inc., Otsu, Japan) according to the manufacturer's protocol.

The expression of $M A P 2 K 7$ mRNA and mature miR$125 \mathrm{~b}$ was determined by quantitative real-time PCR (qPCR) using KAPA SYBR FAST Universal qPCR Kit (KK4601; Kapa Biosystems, Wilmington, MA, USA) according to the manufacturer's protocol, and $18 \mathrm{~S}$ and U6 were taken as internal controls for $M A P 2 K 7$ and miR$125 \mathrm{~b}$, respectively. The primer sequences were as follows: MAP2K7 F: 5'-ATGACAGTGGCGATTGTGAAGG-3', R: 5'-CAGGATGTTGGAGGGCTTGAC-3'; $18 \mathrm{~S}$ F: 5'-CAGCCACCCGAGATTGAGCA-3', R: 5'-TAGTAGC GACGGGCGGTGTG-3'; miR-125b F: 5'-GCUCCCUGA GACCCUAAC-3', R: 5'-CAGTGCAGGGTCCGAGGT-3'; U6 F: 5'-CTCGCTTCGGCAGCACATATACT-3', R: 5'-ACGCTTCACGAATTTGCGTGTC-3'. To calculate the expression of miR-125b and $M A P 2 K 7,2^{-\triangle \Delta C T}$ method was used and each sample was run in triplicate.

\section{Western blot}

Cells were harvested in phosphate-buffered saline (PBS) and centrifuged for 1 minute at 13,000 rpm. The pellet was resuspended in $100 \mu \mathrm{L}$ of lysis buffer (radio-immunoprecipitation assay [Beyotime, Shanghai, People's Republic of China], Cocktail, and phenylmethanesulfonyl fluoride [Hoffman-La Roche Ltd., Basel, Switzerland]) and incubated for 30 minutes on ice. The lysate was centrifuged for 10 minutes at $12,000 \mathrm{rpm}$ at $4^{\circ} \mathrm{C}$. The protein amount was determined using the bovine serum albumin (BSA) kit (Beyotime). For immunoblotting, the following antibodies were used: anti-MAP2K7 (55030-1-Ap; Proteintech, Rosemont, IL, USA), anti-E-cadherin (ab1416; Abcam, Cambridge, UK), anti-N-cadherin (4061; cell signaling technology), and anti-GAPDH (AP0063; Bioworld Technology, St Louis Park, MN, USA). Bound antibodies were detected by using peroxidase-labeled secondary antibodies (Proteintech) and immunoblotting and enhanced chemiluminescence detection reagents from Thermo Fisher Scientific.

\section{Wound healing assay}

Migration of transfected Hs578T cells was detected by a wound healing assay. Six-well plates were used in this experiment and each well was seeded with transfected cells to $90 \%$ confluence. Cells were maintained at normal conditions for 12 hours to permit cell adhesion, and a scratch was made manually by scraping the cell monolayer with $200 \mu \mathrm{L}$ pipette tip. Then, the concentration of FBS in the cell culture medium was changed from $10 \%$ to $1 \%$ immediately. Cells in each well were captured under a microscope at different time points. All the scratch assays were performed in quadruplicate.

\section{Transwell assay}

For transwell invasion assay, the matrigel (BD, Franklin Lakes, NJ, USA) was diluted 1:4 with serum-free medium, and the membrane was coated with matrigel to form a matrix barrier. Then, $5 \times 10^{4}$ cells were suspended in $200 \mu \mathrm{L}$ serum-free medium with $0.1 \% \mathrm{BSA}$ and placed in the upper chamber. In each lower chamber, $500 \mu \mathrm{L}$ medium with $15 \%$ FBS was added. For migration assay, the cells were incubated for 24 hours, and for invasion assay, the cells were incubated for 48 hours. Cells that had migrated or invaded through the pores were fixed with $95 \%$ ethyl alcohol for 15 minutes and stained with $0.1 \%$ crystal violet for 15 minutes. The cells were then photographed and counted under a microscope.

\section{Immunohistochemistry}

The MAP2K7 protein was studied by immunohistochemistry (IHC). Paraffin-embedded, formalin-fixed tissue was cut into $4 \mu \mathrm{m}$ sections, placed on polylysine-coated slides, deparaffinized in xylene, and rehydrated through a graded ethanol series. Endogenous peroxidase activity was quenched in $3 \%$ hydrogen peroxide, and the tissue was subjected to antigen retrieval treatment by boiling slides for 10 minutes at a high pressure in improved citrate antigen retrieval solution (Beyotime, $\mathrm{pH}$ 6.0). The sections were incubated at $4^{\circ} \mathrm{C}$ overnight with the MAP2K7 antibody (sc-25288; Santa Cruz Biotechnology Inc., Dallas, TX, USA). The immunostaining procedure was performed with the ChemMate DAKO EnVision Detection Peroxidase/DAB kit (Dako Denmark 
A/S, Glostrup, Denmark), which resulted in a brown-colored precipitate at the antigen site. Subsequently, the sections were counterstained with hematoxylin-eosin, dehydrated, hyalinized, and sealed.

\section{Statistical analyses}

Statistical analyses were performed using SPSS version 21.0 (SPSS Inc., Chicago, IL, USA). All data were presented as the mean \pm standard deviation. Values of $P<0.05, P<0.01$, and $P<0.001$ were considered to be statistically significant.

\section{Results}

\section{miR-I 25 b is downregulated in TNBC specimens}

It has been demonstrated that miR-125b is critically involved in the progression of human cancer. To determine whether miR-125b plays a pivotal role in TNBC, we measured the expression of miR-125b in 23 pairs of clinical specimens. Our results revealed that miR-125b was significantly downregulated in 19 TNBC tissues (83\% of total) compared with their corresponding normal tissues (Figure 1), indicating that downregulation of miR-125b is associated with TNBC development and progression.

\section{miR-I25b decreases migration and invasion of TNBC cells}

To investigate the biological effect of miR-125b in TNBC cells, miR-125b precursor was transfected into Hs578T cells, which showed strong migration and invasion abilities, to increase the level of ectopic miR-125b (Figure 2A). We demonstrated that miR-125b not only decreased cell migration (Figure 2B and C) but also led to slow invasion (Figure 2D).

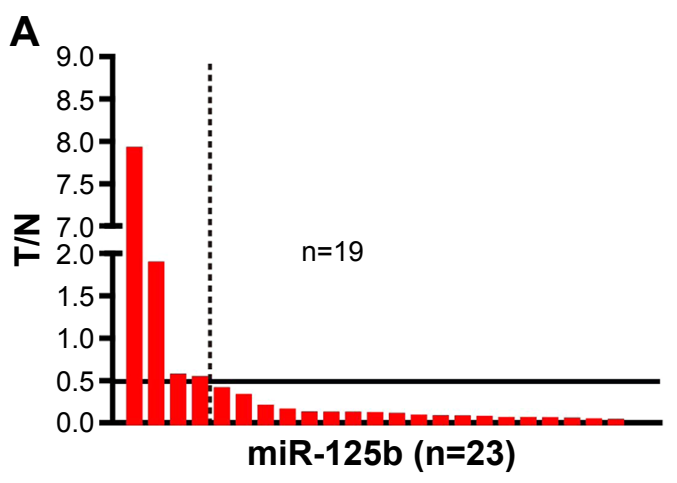

These data suggest that miR-125b is an important tumor suppressor, which exerts its function by inhibiting migration and invasion of TNBC cells.

\section{miR-I 25b targets MAP2K7 and inhibits EMT}

In order to find out the mechanism of action of miR-125b on TNBC cells, TargetScan, PicTar, and miRanda were used for possible targets and $M A P 2 K 7$ was considered to be the putative target of miR-125b (Figure 3A). To verify that miR-125b targets $M A P 2 K 7$, the full-length $M A P 2 K 7$ 3 '-UTR was cloned after Renilla luciferase reporter gene into psiCHECK-2, which also constitutively expresses the Firefly luciferase gene as an internal control. A significant decrease in the Rluc/Fluc ratio was found when cotransfecting with miR-125b but not with the negative control group (NC) (Figure 3B). qPCR demonstrated that upregulation of miR-125b notably reduced the expression of target gene (MAP2K7) and mesenchymal markers ( $\mathrm{N}$-cadherin, vimentin, and fibronectin), but enhanced the expression of epithelial marker E-cadherin (Figure 3C). Furthermore, Western blot also showed decreased MAP2K 7 and N-cadherin, and increased E-cadherin (Figure 3D) expression, suggesting that miR-125b hinders EMT through targeting to $M A P 2 K 7$ 3'-UTR and inhibiting its expression.

\section{MAP2K7 depletion mimics the effect of miR-I25b}

To verify the effect of MAP2K7 on TNBC cells, we knocked down the endogenous expression level of MAP2K7 in Hs578T cells using siRNA. Downregulation of MAP2K7 resulted in less migration and invasion compared with the NC group (Figure 4A-C), indicating MAP2K7 is associated

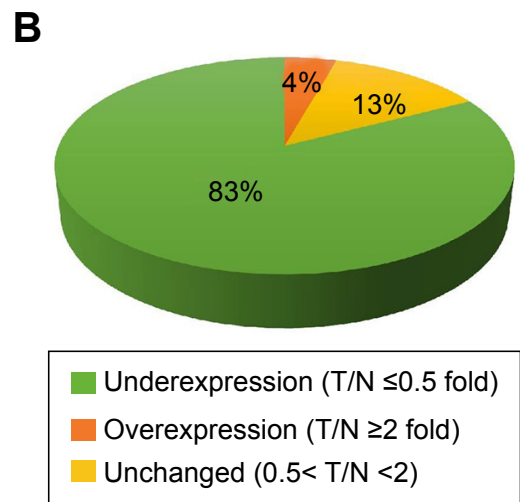

Figure I miR-125b is downregulated in TNBC tissues.

Notes: (A) The figure shows fold changes of the relative expression of miR-125b (T/N). miR-125b expression in TNBC samples (T, $n=23)$ and adjacent breast cancer tissues

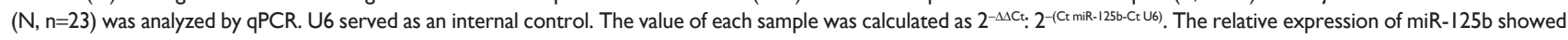
underexpression (T/N $\leq 0.5$ fold) in 19 of the TNBC tissues. (B) The figure shows the distribution of miR-I25b expression in clinical specimens. miR- $125 \mathrm{~b}$ relative expression showed underexpression ( $\mathrm{T} / \mathrm{N} \leq 0.5$ fold) in $83 \%$ of the TNBC tissues, overexpression (T/N $\geq 2$ fold) in $4 \%$ of the TNBC tissues, and was unchanged $(0.5<\mathrm{T} / \mathrm{N}<2$ ) in others. Vertical dotted line in $(\mathbf{A})$ stands for separatrix $(\mathrm{T} / \mathrm{N}=0.5)$. Nineteen pairs had a ratio below 0.5 -fold (right of the vertical dotted line), and the others had a ratio above 0.5 -fold (left of the vertical dotted line).

Abbreviations: TNBC, triple-negative breast cancer; qPCR, quantitative real-time polymerase chain reaction. 
A

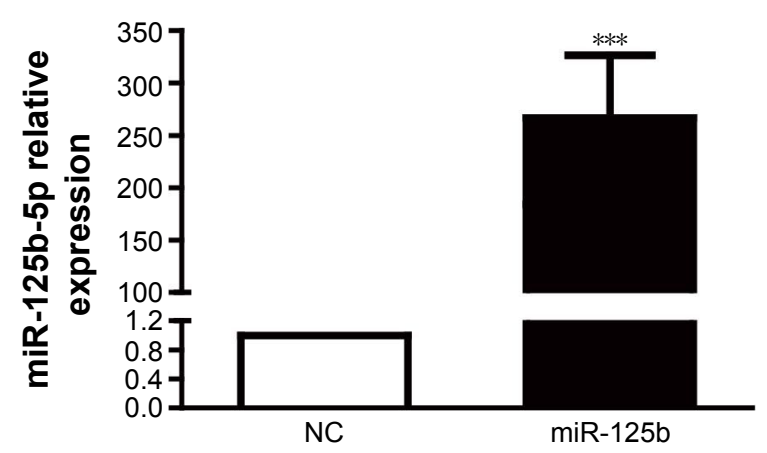

C
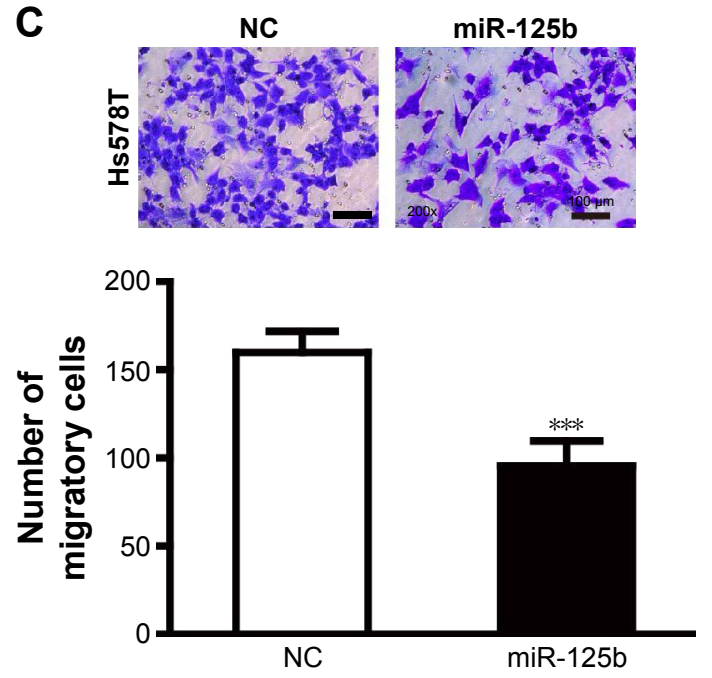

B
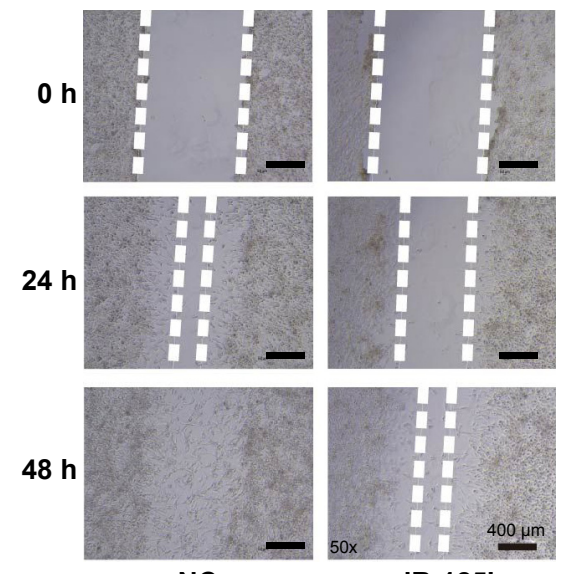

D

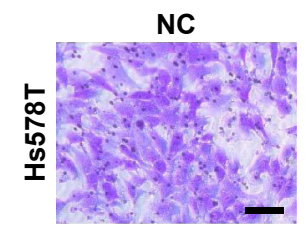

miR-125b

miR-125b
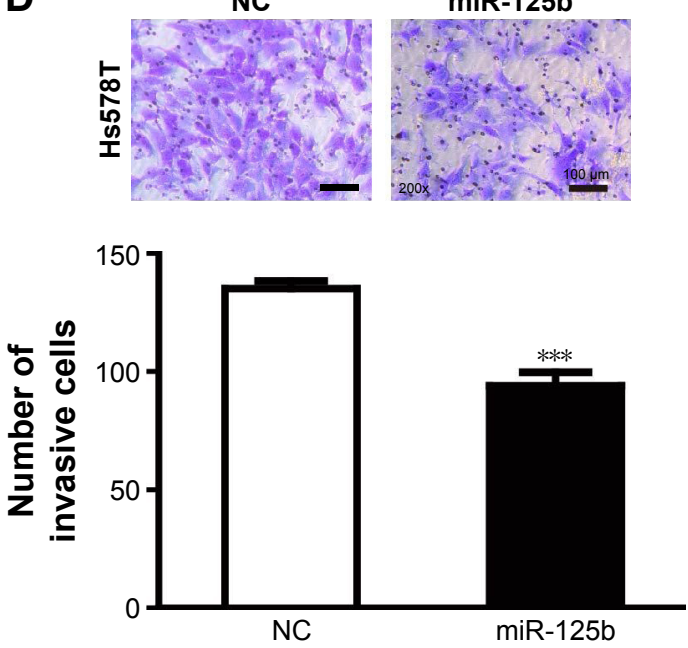

Figure 2 miR-125b suppresses migration and invasion of TNBC cells.

Notes: (A) The figure shows miR-125b relative expression in Hs578T cells expressing miR-125b. U6 was used as an internal control. The value of each sample was calculated as $2^{-\Delta \Delta C t}$ and Student's $t$-test was used. Data were present as mean $\pm S D, n=3$, and $* * * p<0.00$ I vs control group (NC). (B) Wound healing assay was used to detect the motility in Hs578T cells expressing miR-I25b. Movement of Hs578T cells into the wound was shown for mimics-transfected and untransfected cells. Representative images $(\times 50)$ at 0,24 , and 48 hours are given. (C) Shows transwell migration assay. miR-125b overexpression and control-transfected Hs578T cells plated on the transwell migration chambers were allowed to migrate through $8 \mu \mathrm{m}$ pores for 24 hours. A total of three membranes were used for each sample. Representative images $(\times 200)$ are given above the graph. Student's $t$-test was used with $* * * P<0.00 \mathrm{I}$. (D) Shows transwell invasion assay. miR-I25b overexpression and control-transfected Hs578T cells were allowed to invade through $8 \mu \mathrm{m}$ pores for 36 hours. A total of three membranes were used for each sample. Representative images $(\times 200)$ are given above the graph. Student's $t$-test was used with $* * * P<0.001$.

Abbreviations: TNBC, triple-negative breast cancer; SD, standard deviation; $h$, hours; NC, negative control.

with migration and invasion of TNBC cells. Moreover, MAP2K7 depletion led to increased expression of E-cadherin and decreased expression of $\mathrm{N}$-cadherin, vimentin, and fibronectin, which was consistent with the results obtained in the Western blot (Figure 4D and E) analyses. These data suggest that MAP2K7 depletion inhibits EMT, which mimics the effect of miR-125b.

\section{MAP2K7 overexpression partly rescues the EMT suppression of miR-125b}

To further determine the function of MAP2K7 on TNBC cells, the overexpression plasmid MAP2K7-pcDNA3.1(-) was constructed and cotransfected with miR-125b into Hs578T cells.
The results showed that overexpression of MAP2K 7 in miR125b-expressing Hs $578 \mathrm{~T}$ cells partly rescued the migration and invasion inhibited by miR-125b (Figure 5A and B). Moreover, the expression of E-cadherin, N-cadherin, vimentin, and fibronectin in MAP2K7-overexpressing Hs578T cells was consistent with $\mathrm{NC}$ group but significantly differed from miR-125b-expressing cells (Figure 5C and D), indicating that MAP2K7 partly reversed the effect of miR-125b.

\section{MAP2K7 expression is associated with TNBC}

We examined the expression of MAP2K7 in TNBC and normal tissues using IHC. We found that MAP2K7 is overexpressed 
A
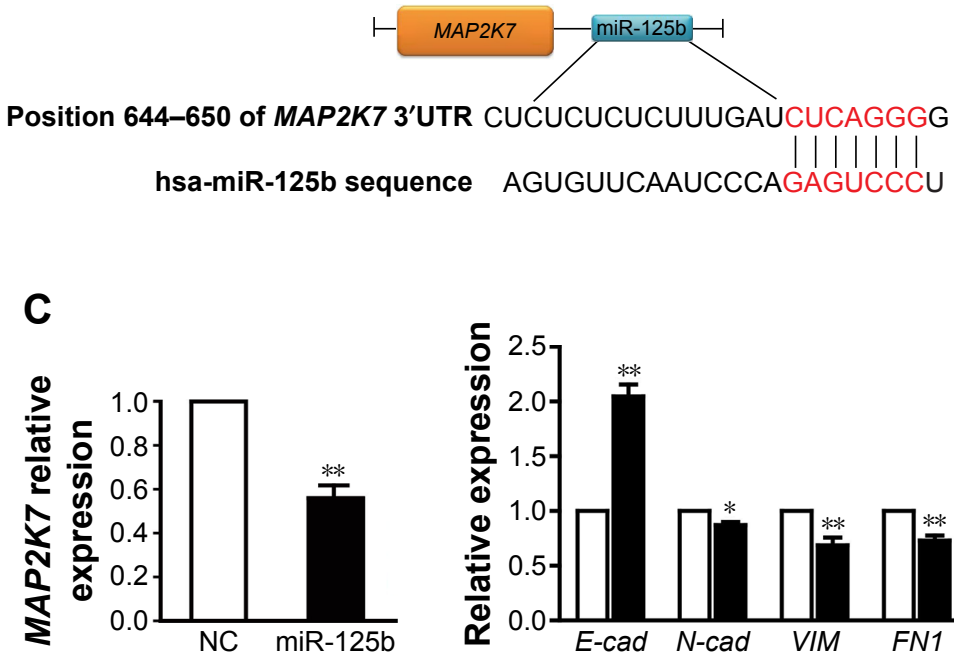

B

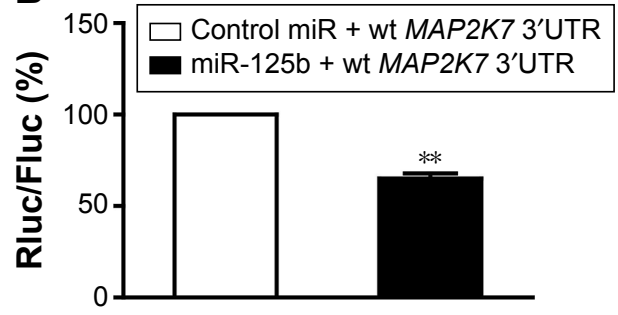

D

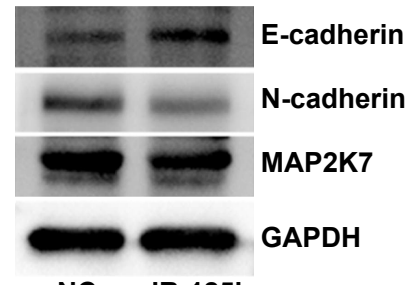

NC miR-125b

NC $\square$ miR-125b

Figure 3 miR-125b targets 3'UTR of MAP2K7 and hinders EMT.

Notes: (A) The binding site between miR-125b and MAP2K7 3'UTR was identified by TargetScan. The region of the MAP2K7 3'UTR that interacted with miR-I25b is highlighted in red. (B) 293T cells were transfected with Wt reporter plasmid and miR-125b or control. After transfection for 48 hours, luciferase activity was measured by a dual luciferase reporter assay. The result is presented as Rluc/Fluc\% (Renilla/Firefly luciferase), the Rluc/Fluc\% of control group is set as $100 \%$. Each bar represents the mean \pm SD of three independent experiments; $* * P<0.0$ I. (C) Relative expression of MAP2K7 and EMT markers (E-cadherin, $N$-cadherin, vimentin, and fibronectin) in Hs578T cells expressing miR-125b compared with control (NC). U6 was used as an internal control for miR-125b and I8S was used for EMT markers. The value of each sample was calculated as $2-\Delta \Delta \mathrm{Ct}$. Student's $t$-test was used. ${ }^{*} P<0.05$; ${ }^{*} * \mathrm{P}<0.0 \mathrm{I}$. (D) Expression of E-cadherin, N-cadherin, and MAP2K7 by Western blot with got function of miR-I25b. GAPDH served as internal reference.

Abbreviations: EMT, epithelial-mesenchymal transition; UTR, 3'-untranslated region; SD, standard deviation; E-cad, E-cadherin; N-cad, N-cadherin; VIM, vimentin; FNI, fibronectin.

A

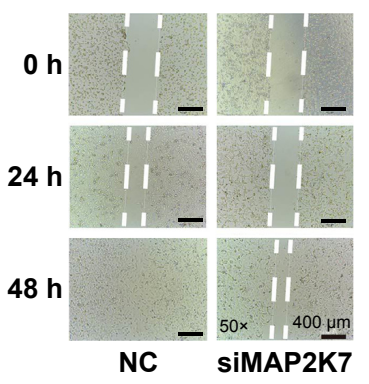

D

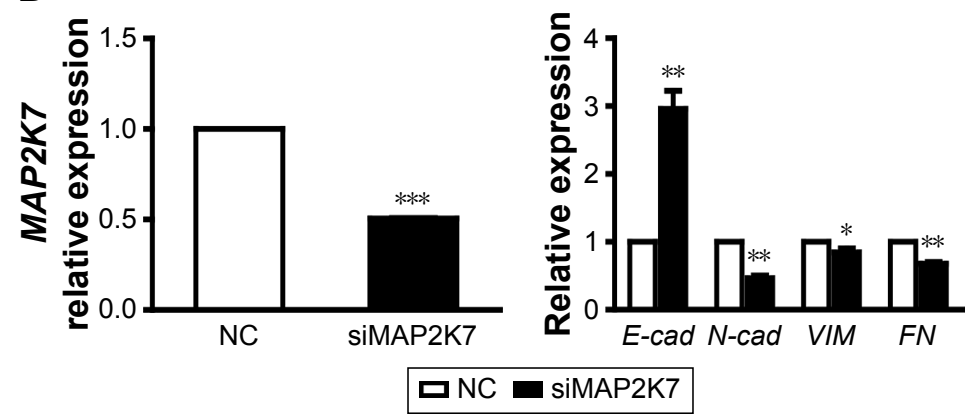

B
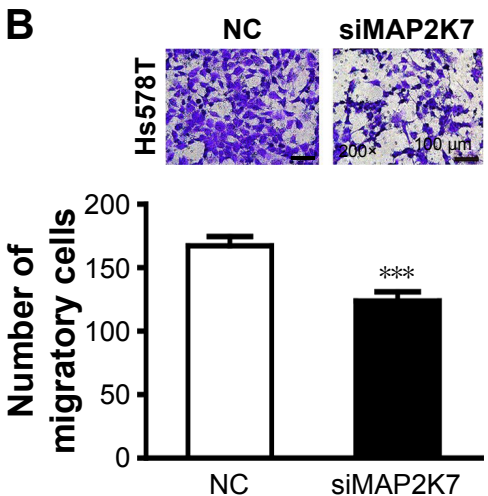

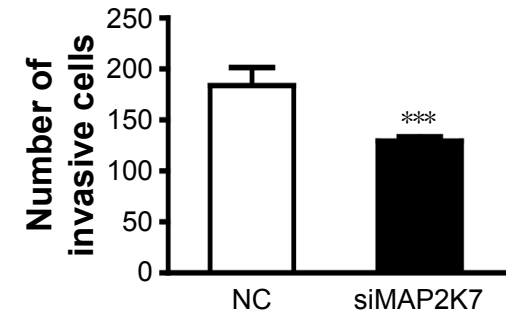

$\mathbf{E}$

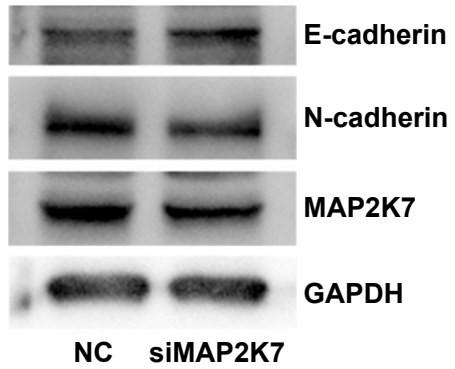

Figure 4 MAP2K7 depletion mimics the effect of miR-I25b.

Notes: (A-C) Hs578T cells were transfected with siMAP2K7 or negative control (NC) and then processed with (A) wound healing assay, (B) transwell migration assay, and (C) transwell invasion assay; $* * * P<0.001$. (D, E) The expression of MAP2K7 and EMT markers with lost function of MAP2K7 in Hs578T cells. (D) I8S was used as an internal control for EMT markers. Each bar represents the mean \pm SD of three independent experiments; $* P<0.05 ; * * P<0.0$; $* * * P<0.00 I$. (E) GAPDH served as internal reference. Abbreviations: EMT, epithelial-mesenchymal transition; SD, standard deviation; E-cad, E-cadherin; N-cad, N-cadherin; VIM, vimentin; FNI, fibronectin; h, hours. 

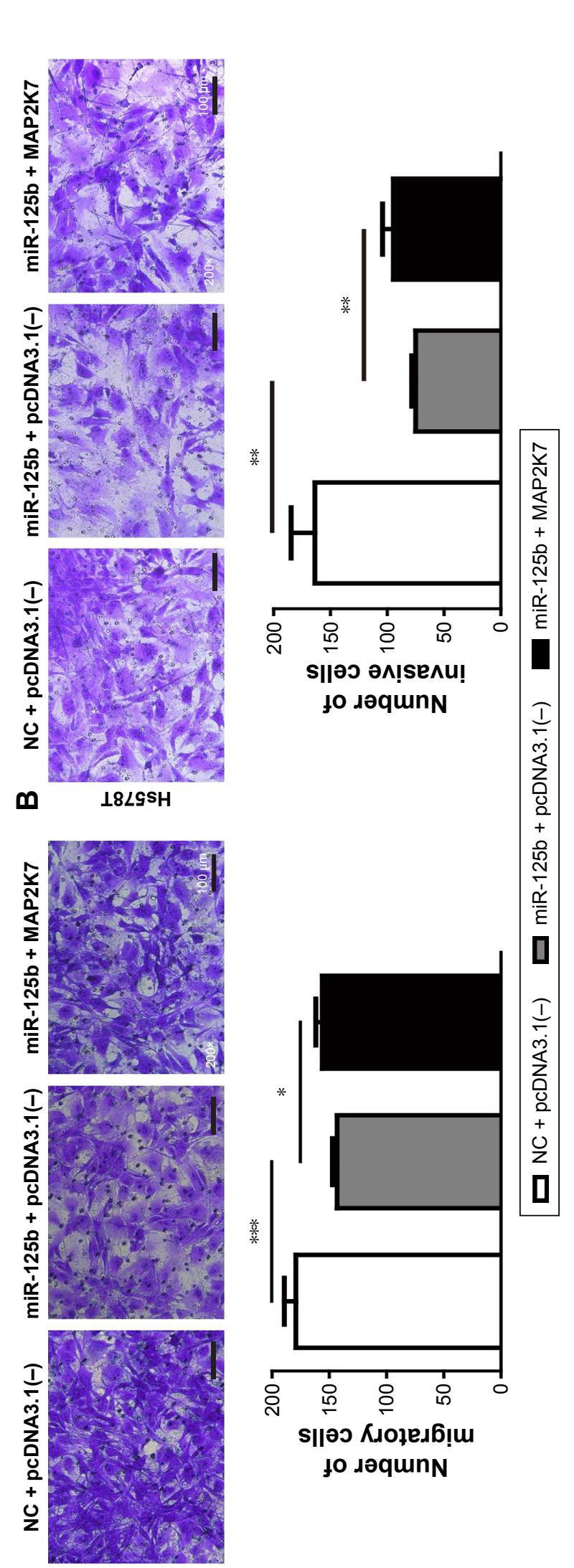

18LGSH

$<$

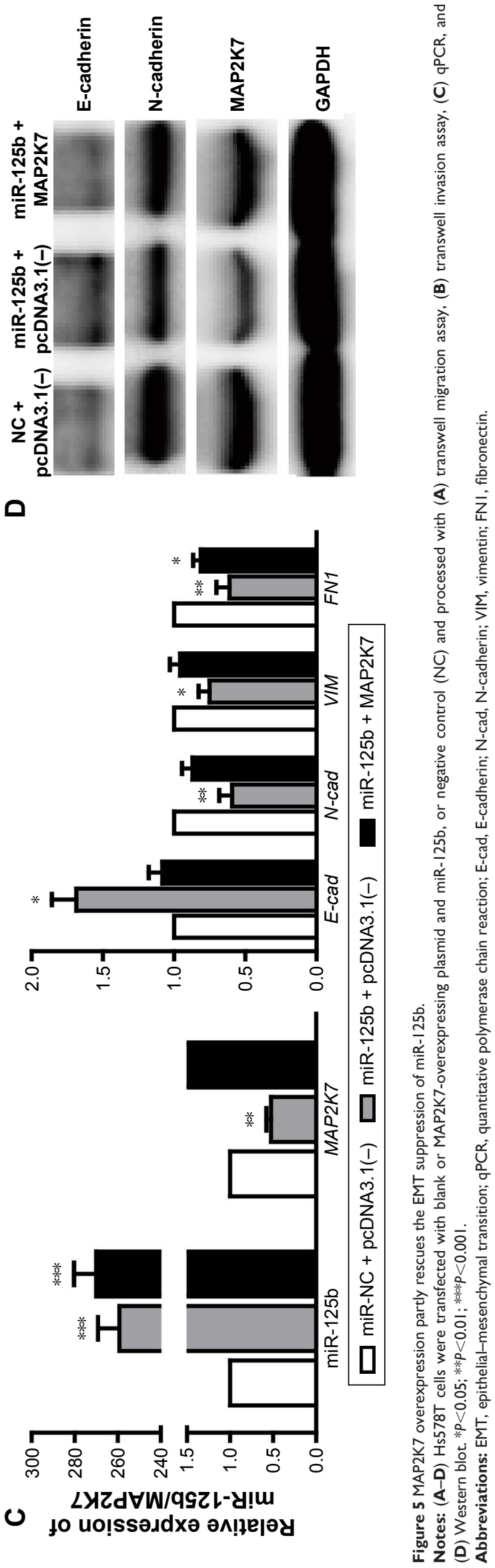


A

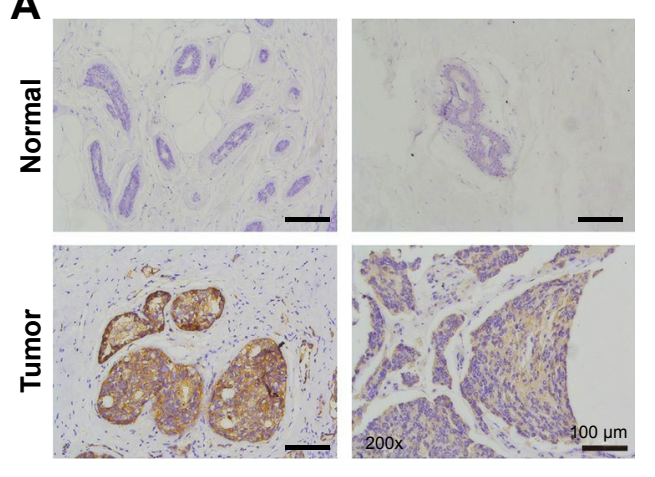

B

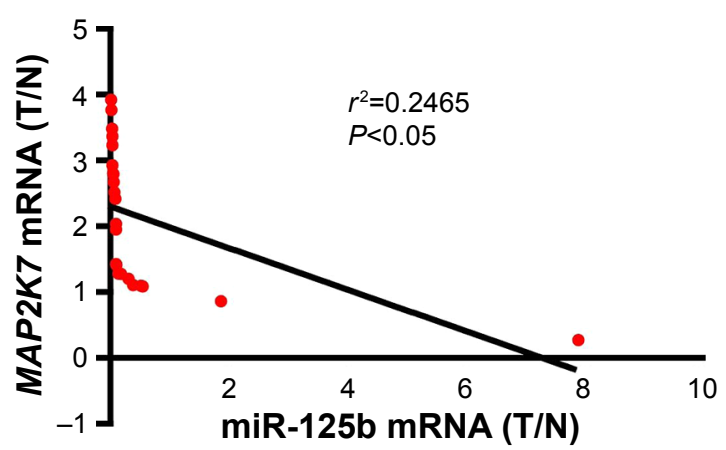

Figure 6 The correlation between MAP2K7 and miR-125b in TNBC specimens.

Notes: (A) Shows the expression of MAP2K7 in clinical specimens by immunohistochemistry. Representative IHC photos (200x) of MAP2K7 expression in normal and TNBC specimens are presented. MAP2K7 staining was mainly localized within the cytoplasm of cells in the form of yellow brown granules. All sections were counterstained with hematoxylin and eosin. (B) Shows negative correlation between the expression of miR-125b and MAP2K7 in 23 TNBC samples $\left(r^{2}=0.2465\right.$; $\left.P<0.05\right)$. The relative expression of miR-125b and MAP2K7 was quantified using QPCR and normalized by U6 or I8S.

Abbreviations: TNBC, triple-negative breast cancer; IHC, immunohistochemistry; qPCR, quantitative polymerase chain reaction; N, normal; T, tumor.

in cytoplasm of TNBC cells but scarcely expressed in normal breast tissues (Figure 6A). We further analyzed the expression of MAP2K 7 and miR-125b in clinical specimens with Pearson correlation analysis which showed an inverse correlation between them (Figure 6B; $r^{2}=0.2465, P<0.05$ ).

\section{Discussion}

TNBCs have an increased propensity to metastasize, and the majority of deaths from this disease are a result of distant disease. EMT has become the focus of research into the metastatic process. Over the last 10 years, there have been multiple studies identifying miRNA changes associated with TNBC. Emerging evidence indicates that miR-125b acts as a double-faced gene expression regulator, described both as a tumor suppressor and an oncogene in multiple cancer types. ${ }^{18-20}$ Its expression was found to be downregulated in a variety of solid cancers, including lung cancer, hepatic cancer, thyroid cancer, ovarian cancer, cervical cancer, melanoma, and neuroblastoma, but upregulated in gastric and pancreatic cancers. ${ }^{18}$ Previous research showed that miR-125b was downregulated and associated with poor prognosis and chemoresistance in TNBC, ${ }^{21}$ but the mechanism involved is still unknown, so our study focused on determining the effect of miR-125b on TNBC. In our study, we chose 23 pairs of TNBC tissues for study and indicated that 19 of 23 TNBC samples showed downregulated miR-125b compared with corresponding normal tissues, suggesting that miR-125b plays an important role in TNBC. Then, functional research of miR-125b was conducted, which confirmed that miR-125b inhibited EMT of Hs578T cells in vitro.

As a result of the potential of one miRNA to target multiple gene transcripts, many targets have been identified to participate in the process of breast cancer which is modulated by miR-125b. miR-125b conferred chemoresistant phenotype by targeting $\mathrm{Mcl}-1$ or $\mathrm{Bakl},{ }^{22,23}$ controlled clinically relevant cancer features by inhibiting $E P O$ and $E P O R,{ }^{18}$ and regulated migration and invasion through binding with $M M P 13$ or ARID $3 B .{ }^{24}$ Although the molecular mechanism of EMT suppression caused by miR-125b in the Hs578T cells was still unclear in our study, we speculate that miR-125b can target MAP $2 K 7$ to regulate EMT by using computational tools for miRNA target prediction, which was confirmed by dual luciferase assay.

We further investigated whether the effect of miR-125b on EMT was MAP2K7-dependent through knockdown of MAP2K7 or rescue of MAP2K7 in miR-125b-expressing cells. Our data showed that knockdown of MAP2K 7 mimics the effects of miR-125b overexpression which leads to the EMT inhibition in Hs578T cells. Furthermore, MAP2K7 overexpression reversed the inhibitory effect of miR-125b on migration and invasion of Hs578T cells. In order to seek the clinical relevance, we also evaluated the expression level of MAP2K7 in TNBC tissues and found that its expression is upregulated in TNBC and negatively correlated with the level of miR-125b.

Two MAPK kinases (MKK4 and MKK7) can activate the JNK group of MAPK in vitro. JNK is phosphorylated preferentially on Tyr by MKK4 and on Thr by MKK $7 .{ }^{17}$ MAP2K7 is able to specifically activate the JNK pathway without affecting ERK and p38 activities. ${ }^{14,16}$ This selectivity differs from that of MKK4, which is capable of activating the JNK and p38 subgroups. ${ }^{25} \mathrm{MAP} 2 \mathrm{~K} 7$, as well as its downstream kinase JNK, is reported to play a context-dependent and cell type-specific role in cancer 
development. ${ }^{26}$ Previous researches indicated that high level of MAP2K7 is observed in colon, hepatocellular, and breast cancers. ${ }^{27}$ Activated MAP2K7 enhanced the proliferation, metastasis, and progress of cancer. ${ }^{27-29}$ Moreover, it was associated with DNA damage response scenery. ${ }^{30}$ Specifically, MAP2K7 was demonstrated to significantly delay the onset of early-stage tumors, as well as to impede the progression of malignancy in mouse models of lung and mammary cancer driven by KRasG12D and NeuT overexpression, respectively. ${ }^{30}$ As the downstream of MAP2K7, JNK expression in tumor cells integrates stromal signals to promote tumor cell invasion. Once tumor cells enter the bloodstream, JNKs increase circulating tumor cell (CTC) survival and homing to tissues. By promoting fibrosis, JNKs improve CTC attachment to the endothelium. Once anchored, JNKs stimulate EMT to facilitate tumor cell extravasation and enhance the secretion of endothelial barrier disrupters. ${ }^{31}$ So we speculate that miR-125b suppressed EMT through JNK pathway which was modulated by MAP2K 7 activation. In future, the potential importance of the MAP2K7-JNK cascade for the prevention of EMT in TNBC will be evaluated.

\section{Conclusion}

In conclusion, this study identifies miR-125b as a novel potential tumor suppressor in TNBC by targeting MAP2K7, which is the upstream activator of JNK. However, the potential importance of the MAP2K7-JNK cascade in miR-125bmediated EMT in TNBC cells remains to be clarified. Thus, much more work is still required to determine its detailed functional mechanisms in TNBC and the potentiality of miR-125b as a therapeutic target for TNBC.

\section{Acknowledgment}

This work was supported by Hangzhou Science and Technology Development Plans (20130733Q37, 20090833B08).

\section{Author contributions}

Liquan Hong: conception and design, acquisition of data, analysis, and interpretation of data, and draft the article; Feng Pan, Huifen Jiang, Lahong Zhang, Yuhua Liu, Chengsong Cai, Chunzhen Hua, and Xian Luo: acquisition and analysis of data; Jinhua Sun: acquisition of data, analysis, and interpretation of data; Zhaojun Chen: conception and design, revised the article. All authors contributed toward drafting and revising the paper and agree to be accountable for all aspects of the work.

\section{Disclosure}

The authors report no conflicts of interest in this work.

\section{References}

1. Wang H, Tan G, Dong L, et al. Circulating MiR-125b as a marker predicting chemoresistance in breast cancer. PLoS One. 2012;7(4):e34210.

2. Chen L, Yang L, Qiao F, et al. High levels of nucleolar spindle-associated protein and reduced levels of BRCA1 expression predict poor prognosis in triple-negative breast cancer. PLoS One. 2015;10(10):e0140572.

3. LaBarge MA, Mora-Blanco EL, Samson S, Miyano M. Breast cancer beyond the age of mutation. Gerontology. Epub 2015 Nov 6.

4. Liu Y, Zhu P, Wang Y, et al. Antimetastatic therapies of the polysulfide diallyl trisulfide against triple-negative breast cancer (TNBC) via suppressing MMP2/9 by blocking NF-kappaB and ERK/MAPK signaling pathways. PLoS One. 2015;10(4):e0123781.

5. Navratil J, Fabian P, Palacova M, Petrakova K, Vyzula R, Svoboda M. Triple negative breast cancer. Klin Onkol. 2015;28(6):405-415. Czech.

6. O'Reilly EA, Gubbins L, Sharma S, et al. The fate of chemoresistance in triple negative breast cancer (TNBC). BBA Clin. 2015;3:257-275.

7. Hofmann MH, Heinrich J, Radziwill G, Moelling K. A short hairpin DNA analogous to miR- $125 \mathrm{~b}$ inhibits C-Raf expression, proliferation, and survival of breast cancer cells. Mol Cancer Res. 2009;7(10):1635-1644.

8. Saetrom P, Biesinger J, Li SM, et al. A risk variant in an miR-125b binding site in BMPR1B is associated with breast cancer pathogenesis. Cancer Res. 2009;69(18):7459-7465.

9. Chen X, Liu J, Feng WK, Wu X, Chen SY. MiR-125b protects against ethanol-induced apoptosis in neural crest cells and mouse embryos by targeting Bak 1 and PUMA. Exp Neurol. 2015;271:104-111.

10. Tan L, Yu JT, Liu QY, et al. Circulating miR-125b as a biomarker of Alzheimer's disease. J Neurol Sci. 2014;336(1-2):52-56.

11. Mar-Aguilar F, Luna-Aguirre CM, Moreno-Rocha JC, et al. Differential expression of miR-21, miR-125b and miR-191 in breast cancer tissue. Asia-Pacific J Clin Oncol. 2013;9(1):53-59.

12. Mathe A, Scott RJ, Avery-Kiejda KA. MiRNAs and other epigenetic changes as biomarkers in triple negative breast cancer. Int JMol Sci. 2015; 16(12):28347-28376.

13. Komagata S, Nakajima M, Takagi S, Mohri T, Taniya T, Yokoi T. Human CYP24 catalyzing the inactivation of calcitriol is post-transcriptionally regulated by miR-125b. Mol Pharmacol. 2009;76(4):702-709.

14. Wang Y, Su B, Sah VP, Brown JH, Han J, Chien KR. Cardiac hypertrophy induced by mitogen-activated protein kinase kinase 7, a specific activator for c-Jun NH2-terminal kinase in ventricular muscle cells. J Biol Chem. 1998;273(10):5423-5426.

15. Zhu T, Wang J, Pei Y, et al. Neddylation controls basal MKK7 kinase activity in breast cancer cells. Oncogene. Epub 2015 Sep 14.

16. Lu J, Liu Z, Zhao L, Tian H, Liu X, Yuan C. In vivo and in vitro inhibition of human liver cancer progress by downregulation of the mu-opioid receptor and relevant mechanisms. Oncol Rep. 2013;30(4):1731-1738.

17. Tournier C, Dong C, Turner TK, Jones SN, Flavell RA, Davis RJ. MKK7 is an essential component of the JNK signal transduction pathway activated by proinflammatory cytokines. Genes Dev. 2001;15(11): 1419-1426.

18. Ferracin M, Bassi C, Pedriali M, et al. miR-125b targets erythropoietin and its receptor and their expression correlates with metastatic potential and ERBB2/HER2 expression. Mol Cancer. 2013;12(1):130.

19. Jiang $\mathrm{F}$, Liu $\mathrm{T}, \mathrm{He} \mathrm{Y}$, et al. MiR-125b promotes proliferation and migration of type II endometrial carcinoma cells through targeting TP53INP1 tumor suppressor in vitro and in vivo. BMC Cancer. 2011; 11:425.

20. Feliciano A, Castellvi J, Artero-Castro A, et al. miR-125b acts as a tumor suppressor in breast tumorigenesis via its novel direct targets ENPEP, CK2-alpha, CCNJ, and MEGF9. PLoS One. 2013;8(10): e76247.

21. Ouyang M, Li Y, Ye S, et al. MicroRNA profiling implies new markers of chemoresistance of triple-negative breast cancer. PLoS One. 2014;9(5):e96228. 
22. Zhou M, Liu Z, Zhao Y, et al. MicroRNA-125b confers the resistance of breast cancer cells to paclitaxel through suppression of pro-apoptotic Bcl-2 antagonist killer 1 (Bak1) expression. J Biol Chem. 2010;285(28): 21496-21507.

23. Wang HJ, Guo YQ, Tan G, et al. miR-125b regulates side population in breast cancer and confers a chemoresistant phenotype. J Cell Biochem. 2013;114(10):2248-2257.

24. Wu D, Ding J, Wang L, et al. microRNA-125b inhibits cell migration and invasion by targeting matrix metallopeptidase 13 in bladder cancer. Oncol Lett. 2013;5(3):829-834.

25. Deacon K, Blank JL. MEK kinase 3 directly activates MKK6 and MKK7, specific activators of the p38 and c-Jun NH2-terminal kinases. J Biol Chem. 1999;274(23):16604-16610.

26. Sakai H, Sato A, Aihara Y, et al. MKK7 mediates miR-493-dependent suppression of liver metastasis of colon cancer cells. Cancer Sci. 2014; 105(4):425-430.
27. Guo Y, Wang W, Wang J, et al. Receptor for activated C kinase 1 promotes hepatocellular carcinoma growth by enhancing mitogen-activated protein kinase kinase 7 activity. Hepatology. 2013;57(1):140-151.

28. Marino N, Collins JW, Shen C, et al. Identification and validation of genes with expression patterns inverse to multiple metastasis suppressor genes in breast cancer cell lines. Clin Exp Metastasis. 2014;31(7):771-786.

29. Zubor P, Hatok J, Moricova P, et al. Gene expression abnormalities in histologically normal breast epithelium from patients with luminal type of breast cancer. Mol Biol Rep. 2015;42(5):977-988.

30. Kotsinas A, Papanagnou P, Galanos P, et al. MKK7 and ARF: new players in the DNA damage response scenery. Cell Cycle. 2014;13(8): 1227-1236.

31. Ebelt ND, Cantrell MA, Van Den Berg CL. c-Jun N-terminal kinases mediate a wide range of targets in the metastatic cascade. Genes Cancer. 2013;4(9-10):378-387.
OncoTargets and Therapy

\section{Publish your work in this journal}

OncoTargets and Therapy is an international, peer-reviewed, open access journal focusing on the pathological basis of all cancers, potential targets for therapy and treatment protocols employed to improve the management of cancer patients. The journal also focuses on the impact of management programs and new therapeutic agents and protocols on

\section{Dovepress}

patient perspectives such as quality of life, adherence and satisfaction. The manuscript management system is completely online and includes a very quick and fair peer-review system, which is all easy to use. Visit http://www.dovepress.com/testimonials.php to read real quotes from published authors. 